\title{
La Santa Cruz de Huatulco, elemento sociocultural turístico
}

\section{The Santa Cruz de Huatulco, sociocultural tourist element}

\author{
LÓPEZ-HERNÁNDEZ, Sonia†** \& GARDUÑO-FÉLIX, Gabriel \\ Instituto de Ciencias de la Comunicación, Universidad del Mar, campus Huatulco, Oaxaca México.
}

ID $1^{\text {er }}$ Autor: Sonia, López-Hernández / ORC ID: 0000-0002-9669-1488, Researcher ID Thomson: S-6911-2018

ID $1^{\mathrm{er}}$ Coautor: Gabriel, Garduño-Félix / ORC ID: 0000-0001-5928-8908

DOI: $10.35429 / J O C S .2019 .21 .6 .19 .27$

Recibido: 25 de Octubre, 2019; Aceptado 28 de Diciembre, 2019

\section{Resumen}

En 1984, se expropia más de 21,000 hectáreas de Santa María Huatulco, incluyendo toda el área costera a favor de FONATUR, como resultado de la tendencia positiva mundial del turismo y que México inicia en la década de 1960 con el desarrollo de los Centros Integralmente Planeados (CIP) para que contribuyeran al desenvolvimiento económico de zonas atrasadas o aisladas del país (Espinosa C., El origen del proyecto turístico Cancún, México. Una valoración de sus objetivos iniciales a 42 años de su nacimiento., 2013). Sin embargo, este modelo de desarrollo turístico de Sol y Playa, trajo a la comunidad desafíos e imposiciones de las regulaciones, nuevas técnicas de subsistencia y cambio de posesión de la tierra; lo que cuestiona e interpela profundamente los métodos, costumbres y, fundamentalmente, la identidad de la comunidad, dejando de lado la importancia de la cultura local que no se subsumió al interés económico sino se ignoró. Esta investigación descriptiva se realizó un cruce de técnicas en diferentes niveles: en primer nivel las fuentes de datos que se procesaron en el análisis no tuvieron únicamente un origen disciplinar. Se atendieron diferentes instituciones como son religiosas, históricas y turísticas. El trabajo de recolección de datos es cualitativo basado en revisión de archivo hemerobibliográfico, así como en entrevistas semiestructuradas (Quivy, 2004, pág. 185) y observación no participante (Quivy, 2004, pág. 188).

Bahías de Huatulco, Santa Cruz de Huatulco, Desarrollo Huatulco

\begin{abstract}
In 1984, more than 21,000 hectares of Santa María Huatulco were expropriated, including the entire coastal area in favor of FONATUR, as a result of the positive global tourism trend and that Mexico began in the 1960s with the development of the Integrally Planned Centers. (CIP) to contribute to the economic development of backward or isolated areas of the country (Espinosa C., 2013). However, this model of tourism development of Sun and Beach, brought to the community challenges and impositions of regulations, new subsistence techniques and change of ownership of the land; what questions and deeply questions the methods, customs and, fundamentally, the identity of the community, leaving aside the importance of the local culture that was not subsumed to the economic interest but ignored. This descriptive research was carried out by crossing techniques at different levels: first of all, the data sources that were processed in the analysis were not solely of a disciplinary origin. Different institutions were attended as they are religious, historical and tourist. The data collection work is qualitative based on review of the hemerobibliographic file, as well as semi-structured interviews (Quivy, 2004, page 185) and non-participant observation (Quivy, 2004, page 188).
\end{abstract}

Citación: LÓPEZ-HERNÁNDEZ, Sonia \& GARDUÑO-FÉLIX, Gabriel. La sustentabilidad en tiendas de conveniencia de la ciudad industrial en Villahermosa. Tabasco, para el desarrollo de una propuesta. Revista de Sociología Contemporánea. 2019. 6-21: 19-27.

\footnotetext{
* Correspondencia del Autor (correo electrónico: sonilh74@yahoo.com.mx)

$\dagger$ Investigador contribuyendo como primer autor.
} 


\section{Introducción}

La importancia del fenómeno del turismo y la cultura ha suscitado un interés académico y social dado que es el turismo es un agente de cambio en las sociedades, es decir la actividad turística propicia el encuentro entre visitantes y residentes, lo que conduce inminentemente a transformaciones (Ramírez Hernández, Cruz Jiménez, \& Serrano Barquín, 2019) lo cual justifica los esfuerzos de la comunidad académica por comprender los elementos que hacen posible el turismo no sólo de forma conceptual sino reconociendo los impactos del mismo, por ejemplo Pastor Alfonso (2003) y Ascanio (2003). En este contexto, se entiende que la identidad y la cultura no son inmutables sino que se transforman, en estos procesos de alta complejidad humana la interacción puede presentar significados varios dependiendo el caso y pueden ser de reformulación o ruptura (Bokser Liwerant, Identidad, diversidad, pluralismo, 2008).

Las culturas ante el fenómeno del turismo pueden oponer una resistencia ante la presión para adaptar sus elementos a la cultura de masas. Se trata de procesos de reconfiguración y cambio, hibridación, en los cuales cambian los "sistemas de comprensión de la realidad y sus consecuentes redefiniciones de prácticas y hábitos [...]" (Bokser Liwerant, Identidad, diversidad, pluralismo, 2008, pág. 31). Sin embargo, en las sociedades actuales, los turistas llegan a todas partes, el desarrollo técnico, los transportes y la globalización permiten que lo era un lugar remoto se convierta en un destino turístico de masas. "De hecho no existe una sola zona del mundo en donde no se encuentren turistas, en mayor o menor medida." (Ejarque, 2005, pág. 91).

En México, el sector turístico representa un gran impulsor de la economía En los últimos 60 años, el turismo se ha identificado como impulsor del desarrollo nacional, principalmente como generador de divisas y de empleos e impulsor del progreso regional. Aunque muestra dependencia a desarrollos de sol y playa y una sobreoferta en los destinos principales, entre otros problemas. (Mercado Vargas \& Palmerín Cerna, 2012)
El Centro Integralmente Planeado (CIP) Huatulco conformado como parte del modelo económico de desarrollo neoliberal y de incorporación a la globalización, omitió el desarrollo en consideración del bienestar "en términos de vectores de realización y de la capacidad para conseguirlos" (Sen, 1998, pág. 84) lo cual no se mide en índices objetivos de crecimiento acelerado y sostenido del Producto Interno Bruto (PIB), sino por la percepción de los involucrados con respecto a su progreso social y económico.

Es decir, en términos del sector turístico puede considerarse un proceso de desarrollo atendiendo como el eje más importante el rendimiento económico que se pueda extraer del destino (incluidos los bienes culturales); o bien puede atenderse a procesos de desarrollo que "fueran previamente mediatizados por la voluntad específica de los habitantes de un lugar, en función de sus intereses y valores, de manera que el crecimiento final fuera el resultado de la razón de la población autóctona, sometiéndose el visitante $\mathrm{y}$ las industrias que gestionan su periplo, a los parámetros definidos por la comunidad, en uso de su libertad de elección." (Moragues C., 2006).

Esto da cuenta de la pertinencia del análisis del impacto local en situaciones determinas para comprender las modificaciones del entorno en función del turismo, profundizar en cómo sus habitantes han sido capaces, o no, de adaptarse a las nuevas formas y cómo esa relación lleva a los individuos a interiorizar su propio patrimonio y a presentarlo de manera específica de cara a los visitantes.

Este trabajo es un acercamiento a la milenaria festividad de la Santa Cruz de Huatulco, que no tiene lugar en el desarrollo del CIP Huatulco ni como atractivo ni como seña de identidad para el proyecto. Se presenta un esbozo de la historia de la fiesta religiosa que aún perdura y convoca no sólo a la comunidad originaria del destino sino también a adoradores de comunidades cercanas, y la omisión que se hace de esta realidad cultural por los gestores del desarrollo.

En este orden de ideas se presenta el apartado de metodología para explicar el alcance multidisciplinar del caso. 
En El contexto y surgimiento del CIP Huatulco y La historia de la tradición, se explica que el desarrollo turístico ocupa un territorio cultural-religioso valioso de acuerdo con la interpretación que hacen los descendientes de los pobladores más antiguos. En el apartado Omisión de la Cruz se presentan los diversos proyectos de gestión del lugar y el recurrente olvido de la fiesta. Por último, en las conclusiones se reflexiona acerca de la necesidad de preservar la historia de la comunidad receptora del CIP- Huatulco.

\section{Metodología}

Este proceso complejo difícilmente podría atenderse recurriendo a un único modo de análisis, por lo que en esta investigación descriptiva se realizó un cruce de técnicas en diferentes niveles: en primer nivel las fuentes de datos que se procesaron en el análisis no tuvieron únicamente un origen disciplinar. Para el cruce de datos se atendieron diferentes instituciones como son religiosas, históricas y turísticas. El trabajo de recolección de datos fue cualitativo basado en revisión de archivo y hemerobibliográfica, así como en entrevistas semiestructuradas (Quivy, 2004, pág. 185) y observación no participante (Quivy, 2004, pág. 188). La información fue colectada durante 2011 y 2012.

Una segunda vía para caracterizar la tradición de la fiesta de la Veneración de la Santa Cruz de Huatulco, y evaluar su valor culturalsocial y posiblemente turístico, fue la esfera teórica con el cruce de diferentes perspectivas acerca del objeto de estudio: el turismo, área donde se distingue al turista con necesidades de productos; y la economía para entender los supuestos del enfoque económico neoliberal.

\section{El contexto y surgimiento del CIP Huatulco}

México entra a la liberalización de su mercado bajo un paradigma que ya predominaba el proceso internacional de la producción entre países desarrollados desde la segunda posguerra, y que "debilita la centralidad del mercado nacional" (Dabat, 1994, pág. 29) es decir que el espacio de intercambio económico ya no es local, sino en regiones económicas y con alianzas estratégicas transnacionales.
Para solucionar el déficit en la balanza de pagos y promover el desarrollo regional, a finales de la década de 1960, se proponen los polos de desarrollo turístico para la captación de divisas y estimular el desenvolvimiento económico de zonas atrasadas (Espinosa C., 2013,). En ese contexto el Banco de México, bajo la dirección general de Rodrigo Gómez (1952-1970) y Ernesto Fernández Hurtado (1970-1976), apoyados por el Banco Interamericano de Desarrollo (BID), iniciaron el proceso de promoción del turismo como una herramienta para promover el crecimiento. El BID (Espinosa C., El origen del proyecto turístico Cancún, México. Una valoración de sus objetivos iniciales a 42 años de su nacimiento., 2013,), otorgó en 1979 al gobierno mexicano un primer crédito por 17 millones de dólares para la construcción del Centro Integralmente Planeado (CIP) de Cancún.

Posterior, en 1982 el presidente Miguel de la Madrid Hurtado se concentraría en el nacimiento de Huatulco (FONATUR, Fonatur 35 años. Única historia narrada por sus fundadores y protagonistas, 2010), "Todo estaba listo para cumplir las expectativas del Banco Interamericano de Desarrollo (BID) del Gobierno Federal y de los inversionistas. El modelo económico de México a partir de la década de los ochenta tenía por objetivo reinsertar al país en la economía mundial (Rivera R., M.A; Toledo P. A, 1998). Además, Pedro Vázquez Colmenares, gobernador de Oaxaca, sería un buen aliado para el arranque de las obras. (...) Sin embargo, pronto el desarrollo del polo turístico presentaría grandes complicaciones, entre estas áreas: escasas carreteras y viaje de acceso, problemas comunales, resistencia al cambio por parte de los nativos de la zona y de autoridades locales que no veían con buenos ojos la llegada del Fondo y mucho menos la transformación del uso de las tierras." (FONATUR, Fonatur 35 años. Única historia narrada por sus fundadores $y$ protagonistas, 2010). La importancia de estos modelos de desarrollo económico basados en el turismo se puede evidenciar con "la cuantiosa inversión que el Gobierno Federal ha destinado al desarrollo de éstos, la cual, de acuerdo con cifras presentadas por FONATUR al $2010 \mathrm{y}$ considerando a los CIP's de Cancún, Los Cabos, Ixtapa y Huatulco, ascendía a 3,114 millones de dólares" de los cuales corresponden a Huatulco 190.00 mdd. (Cruz O., 2013). 
Esta propuesta (de CIP) asume la generación de recursos económicos en zonas pobres y la disminución de la disparidad regional (Turismo, Comisión Nacional para el Desarrollo de los Pueblos Indígenas) y, en general, en el desarrollo en el sector turístico intervienen múltiples actores que van desde el Estado (que influye en el desarrollo del sector aplicando políticas y desarrollando la infraestructura y la reglamentación) hasta las principales entidades del sector privado. (Figueroa, E.; F. Pérez; L.Godínez, y R. Pérez, 2014). En este contexto el desarrollo del sector turístico en Oaxaca, y del CIP-Huatulco, se justifica ya que de acuerdo con Pipitone (2007) el estado enfrentó el siglo XX como "un contexto en que las oportunidades de progreso en la economía son escasas entre comunidades atrapadas en la red de los comercios criollo-mestizos y oligarquías comerciales vinculadas con el poder político" (Pipitone, 2007, págs. 15-16). Y aunque el Estado ha aprovechado turísticamente la riqueza cultural de sus regiones en una visión idealizada del pasado indígena desde 1950 con el reconocimiento de la Gelaguetza como fiesta de los oaxaqueños (Maldonado Ramírez, 2013). En la construcción del CIP-Huatulco se han pasado por alto procesos históricos tales como la zona arqueoecológica de Copalita, el descubrimiento de un asentamiento precolonial en la Bahía de Cacaluta (Quadratin, 2016), o ser considerado "el puerto más importante del Pacífico novohispano" (Aguilar O, 2016, pág. 40).

\section{La historia de la tradición}

Así, inscrito en una sociedad capitalista de características neoliberales, el proceso de trasformación del espacio para la implantación del CIP trasmutó el pueblo de Santa María Huatulco, generó conflictos entre diferentes instituciones y desplazó a la comunidad para el desarrollo de la infraestructura turística (Talledos S., 2012). Pero, además de estos problemas de posesión de tierra y laboral entre los oriundos en Huatulco ya analizados (Rodiles, López, \& López, 2015) (Talledos S., 2012), se ignoró la tradición de la Veneración de la Santa Cruz de Huatulco, la cual es una manifestación cultural-religiosa de los grupos sociales que ocupan un territorio cultural más extendido del destinado para el CIP.

\footnotetext{
1 "Quahuitl, que significa el madero, del verbo toloa que es hacer reverencia bajando la cabeza, y de la partícula co, que denota lugar". Eulogio Gillow, Apuntes históricos, Imprenta del Sagrado Corazón, México, 1889. Apéndice I, p. 11 (facsimilar de Ediciones Toledo de 1990).

En el caso de la tradición alrededor de la festividad de la Santa Cruz ("el Madero"), la misma se ha visto disminuida con la expropiación para el desarrollo del CIP, en virtud que los espacios con significado cultural son destinados a otras actividades turísticas (hotelería, restaurantes y comercio.) dejando de lado las tradiciones locales de la comunidad de Huatulco.

Esta tradición puede ser entendida como patrimonio cultural concebido como "las obras materiales y no materiales que expresan la creatividad de ese pueblo: la lengua, los ritos, las creencias, los lugares y monumentos históricos, la literatura, las obras de arte y los archivos y bibliotecas" (Arizpe, L., 2011).

Como punto de partida es de considerar que esta tradición es parte de la historia del lugar y tuvo influencia decisiva en la configuración y vida de la comunidad de la Ciudad de Antequera. La Santa Cruz de Huatulco forma parte de las leyendas, mitos e historias acerca de los orígenes y procesos humanos en Huatulco (Matadamas, 2004). El mismo nombre de la comunidad costera que hoy alberga al CIP, está relacionado con esta tradición: Quauhtolco, "lugar donde se adora o se hace reverencia al palo" ${ }^{\text {, y alude a }}$ una antigua adoración hecha por los chontales de la zona "quienes vieron llegar del mar a un hombre barbado abrazado a aquella cruz".

Esta cruz, a la que se le concedió invulnerabilidad milagrosa frente a los ataques de un grupo de corsarios ingleses en 1587 (De Burgoa, 1997), está relacionada directamente con el origen mismo del cristianismo y la antigua predicación de los apóstoles [según algunos autores de la época Antonio de la Calancha, Francisco de Burgoa y Manuel Duarte (Yáñez G.)], aunque otras fuentes remiten su origen a los primeros frailes que predicaron en el Nuevo Mundo. Cualquiera de las dos versiones le concedió un lugar inmejorable e incuestionable en una época en la que la Iglesia tuvo poder económico, ideológico y social "era una de las instituciones más consolidadas de la Europa de los siglos XVI y XVII. Fueron las órdenes religiosas las que nombraron en gran medida los comportamientos morales y éticos de los colonizadores." (Dalton, 2004, pág. 94). 
En 1612, la Santa Cruz de Huatulco traspasó las fronteras de su comunidad y llegó a la capital donde se le consideró "uno de los cultos fundantes de la iglesia de Antequera, Oaxaca debido a la gran devoción hacia esta reliquia cruciforme hallada en Huatulco, cuyo origen se atribuye a santo Tomás Apóstol" (Yáñez G., págs. 1-2). Fue justamente un hijo de conquistadores, el obispo de Antequera Juan de Cervantes (1608-1614) quien, en 1612, determinó el traslado de la reliquia a la catedral de Antequera (Oaxaca) donde le dedicó un santuario (Yáñez G.) lo cual es significativo para la historia de uno de los referentes arquitectónicos de la capital del Estado y evidencia el impacto social en la comunidad. Otras reliquias, o partes de la Cruz original, se esparcieron en el mundo, desde la Ciudad de Puebla y la Ciudad de México, hasta el Vaticano (Yáñez G.).

Los registros de la Veneración de la Santa Cruz de Huatulco datan del siglo XVII en el centro de Oaxaca. En otras regiones a donde se extendió el culto no hay referencias de archivo debido, por ejemplo, al deterioro del papel por cuestiones naturales en zonas costeras; a la devastación por los diferentes eventos históricos que ha tenido México como la piratería o los levantamientos armados como la Revolución, y porque no de todo se dejaba testimonio "Hay circunstancias que como eran bastante sabidas por la población de la época, por los actores históricos de la época, no se veían en la obligación de dejar un testimonio." (Entrevista a Jesús Joel Peña, 2012)

Actualmente la fiesta celebrada anualmente se rige con el calendario religioso y se realiza el primer viernes de cuaresma en la Bahía de Santa Cruz a la cual acuden adoradores de la cabecera municipal y de pueblos aledaños (Los Naranjos Esquipulas, San Marcial Ozolotepec, San Pedro el Alto, Santo Domingo Petapa), estos llegan a la playa para purificarse al bañarse en el mar, adorar al madero y pedirle un ayuda y protección. Los peregrinos, calculados en más de $500^{2}$, desde hace más de dos décadas, veneran un lugar que para ellos es sagrado porque simboliza la Cruz, la cual ya no existe en su forma original, pero que mantiene su esencia y se representa en otra cruz de madera de menor tamaño.

\section{Omisión de la Cruz}

Con estos antecedentes y aún con el énfasis de la UNESCO para proteger la diversidad cultural iniciado al finalizar la Segunda GM, la expropiación de 21 mil hectáreas destinadas al CIP, en 1983, se orientó con la postura económica del BM. Los responsables del fondo en Huatulco destinaron como puerta de entrada de este paraíso a la Bahía de Santa Cruz, que ya existía y donde Fonatur contaba con el respaldo de la gente del poblado cercano La Crucecita, "zona que más tarde se convertiría en el corazón comercial de Huatulco" (FONATUR, Fonatur 35 años. Única historia narrada por sus fundadores y protagonistas, 2010).

El desarrollo del lugar no contempló la fiesta, ni los peregrinos, de tal manera que el puerto no tiene espacios apropiados para resguardo, sanidad y comida de quienes mantienen esta tradición. Los visitantes adoradores tienen que pernoctar en un edificio en obra negra en el que las asociaciones civiles y religiosas les proveen alimento y cuidados sanitarios mínimos. Los ritos realizados por los adoradores no son conocidos por los turistas que buscan sol y playa y por eso los consideran "raros", "feos", y que "ensucian el mar", por ello no los aprecian y según los veneradores son "mal vistos".

Este modelo de desarrollo turístico es más de un modelo de colonización cultural en el que se borra o ignora la comunidad y tiene "la imagen idealizada acerca del turismo moderno en las zonas tropicales de la que México forma parte, posee elementos que atraen al visitante internacional en busca de las tres "S": sun, sea and sex" (Vázquez S, Valente y E. Propin F., 2004, pág. 146)

El CIP tampoco observó la historia del lugar ni a sus pobladores, ni ecológicas de la zona (la zona ecológica decretada ha sido considerada sin éxito (López, 2007)). Huatulco tiene un gran proyecto que no ha terminado de madurar, pero en ninguna etapa se considera un lugar con historia propia. En 2006, a Huatulco le faltaban cuartos, aviones, servicios, hospitales, escuelas y actividades. Su estrategia consistió en realizar un estudio para detectar las fortalezas y debilidades del proyecto, y así sacarlo adelante. (FONATUR, 2010)

\footnotetext{
${ }^{2}$ Según datos de la Asociación de la Santa Cruz, e incluye: niños, mujeres y hombres. 
Con una inversión de 600 millones de pesos, el 25 de marzo de 2008 se anunció el relanzamiento de Huatulco. Con ello las hermosas Bahías vuelven al mapa nacional e internacional, de la mano de la administración del presidente Felipe Calderón Hinojosa y bajo la tutela de Miguel Gómez Mont (FONATUR, 2010). Pero el enfoque del lugar siguió orientado por el desarrollo de un destino turístico que impone un modelo cultural externo y adecuado al público o cliente que desea atraer, pasando por alto la cultura local; justificado desde una perspectiva económica de subsanar desigualdades e incrementar la derrama económica.

El estudio más reciente de la Secretaría de Turismo para detectar fortalezas del sitio (Turismo, 2013) omite la parte cultural pese a mencionar que el trabajo de identificar en cada destino seleccionado, "las claves que permitan revertir las problemáticas y obstáculos encontrados, para liberar la capacidad creativa y la innovación que asegure capitalizar las ventajas comparativas; o dicho de otra manera, para aprovechar con bases sustentables, la extraordinaria dotación de recursos naturales, culturales y vivenciales disponibles a lo largo y ancho del territorio nacional". De forma particular el FODA de las Bahías de Huatulco no observa las cuestiones culturales del lugar como una fortaleza, pero sí considera una oportunidad la "Posibilidad de incorporar las señas de identidad de la cultura oaxaqueña al inventario turístico del destino", lo cual sigue obviando la cultura local.

El desarrollo del CIP Huatulco podría dar espacio en su concepto de la competitividad turística local, entendida como "la capacidad del destino turístico para atraer viajeros e inversión en infraestructura y equipamiento turístico, que impacte en la llegada de visitantes, incremente la ocupación y el gasto promedio turístico, y que permita generar beneficios para los residentes locales, los prestadores de servicios turísticos, además de favorecer la sostenibilidad del patrimonio natural", para incluir los recursos culturales locales no sólo como un recurso exótico que genera derrama económica sino también como parte del desarrollo de la comunidad originaria y local en función del ejercicio de su libertad de elección, e incluso como capital social (Esquivel Ríos \& López Molina, 2018).
Lo anterior implica salvaguardar el patrimonio cultural inmaterial para su continua recreación y transmisión. Entre las iniciativas para salvaguardar el patrimonio cultural inmaterial se cuentan su identificación, documentación, investigación, preservación, promoción, mejora y transmisión, en particular a través de la educación formal y no formal, así como la revitalización de sus diferentes aspectos. (UNESCO, 2016)

\section{Conclusiones}

El turismo no puede usar banalmente un territorio, no debe entenderse turismo sin entender y tomar en cuenta la cultura local. Por ello, el desarrollo de modelos turísticos que atienden únicamente a la mejora de indicadores económicos, ignora procesos socio-culturales y de identidad que representan las comunidades que acogen o les imponen las actividades turísticas. Para el CIP - Huatulco debe ser relevante salvaguardar el patrimonio cultural inmaterial que implica velar porque éste siga formando parte activa de la vida de las generaciones presentes y se transmita a las venideras. (UNESCO; pág 07) No se discute que parte del fin último pueda ser mejorar la calidad de vida de la población poco desarrollada y minimizar la brecha entre la periferia y el centro, pero es imprescindible entender que "la cultura es el gran activo, la riqueza tangible e intangible, de la que son, objetivamente, propietarios indiscutibles sus propios actores y gestores." (Moragues C., 2006)

La Preservación de la tradición de la Veneración de la Santa Cruz a través del rescate de la historia de la Santa Cruz, resulta por demás relevante ya que estamos ante el surgimiento de un nuevo paradigma de turista con necesidades diferentes, esto es, "la aparición de nuevas tendencias motivacionales en la demanda turística, entre las cuales se halla el aumento de la valoración y la búsqueda de todo lo relacionado con la cultura" (Larrosa R., 2003, pág. 77). Ante ello, es preciso abordar la realidad de las localidades receptoras de turismo, lograr promover el respeto por el entorno, su vida cultural y su riqueza histórica.

La oferta turística del CIP- Huatulco no debe quedarse (como ahora) en etapas de desarrollo acultural, ya que corre el riesgo de perder riqueza de antiguas tradiciones y raíces culturales como la de la tradición de la Veneración de la Santa Cruz de Huatulco. 
Ésta fue relevante en la vida socialreligiosa de la Antequera y del puerto de Huatulco, y continua como una tradición religiosa de la comunidad y se extiende a localidades aledañas al CIP. Plantear proyectos turísticos como el CIP Huatulco debe atender la mejora de la derrama económica pero también si se trata de sostenibilidad, de acuerdo con la Organización Mundial del Turismo, tiene que contemplar las necesidades de los turistas de hoy y de las regiones receptoras; proteger y ampliar las oportunidades para el futuro; respetar "la autenticidad sociocultural de las comunidades anfitrionas, conservar sus activos culturales y arquitectónicos y sus valores tradicionales, y contribuir al entendimiento y la tolerancia intercultural".

\section{Referencias}

Aguilar O, M. (2016). Navegar por el pacífico. Relatos e historias en México, 38-43.

Alva de la Selva, A. (2015). Telecomunciación y TIC en México. España: Comunicación Social Ediciones y Publicaciones.

Arizpe, L. (2006). Los debates internacionales en torno al patrimonio cultural inmaterial. Cuicuilco, 13-27.

Arizpe, L. (2011). Compartir. El patrimonio cultural inmaterial: narrativas y representaciones. México: UNAM-CRIM Consejo Nacional para la Cultura y las Artes.

Contreras, José, María Segovia y María del Mar Camacho. (2013). Origen del endeudamiento en empresas mexicanas y su entrada en concurso mercantil-propuesta de deteccción anticipada de la insolvencia. En M. Palma, Desarrollo Económico en el crecimiento empresarial (págs. 23-38). Bolivia: Universidad Mayor, Real Pontificia de San Francisco De Chuquisaca.

Cruz O., R. (05 de junio de 2013). Centro de Prospectiva y Debate. Obtenido de Centro de Prospectiva y Debate: http://www.contorno.org.mx/articulos/documen to/861/centros-integralmente-planeados-motorde-desarrollo-del-turismo-en-mexico

Dabat, A. (1994). México y la globalización. México: CRIM-UNAM.

Dalton, M. (2004). Breve historia de Oaxaca (Primera ed.). México: FCE.
De Burgoa, F. (1997). Geográfica Descripción de la parte septentrional, del polo Ártico de la América. Edición Facsimiliar. México: Porrúa.

Entrevista a Jesús Joel Peña, m. e. (18 de mayo de 2012). Sobre la Santa Cruz de Huatulco. (S. L. Hernández, Entrevistador)

Espinosa C., H. (ene/jun de 2013). El origen del proyecto turístico Cancún, México. Una valoración de sus objetivos iniciales a 42 años de su nacimiento. LiminaR, 11(1).

Espinosa C., H. (ene/jun de 2013,). El origen del proyecto turístico Cancún, México. Una valoración de sus objetivos iniciales a 42 años de su nacimiento. LiminaR, 11(1).

Espinosa Coria, H. (2013). El origen del proyecto turístico Cancún, México. Una valoración de sus objetivos iniciales a 42 años de su nacimiento.

Esquivel Ríos, R., \& López Molina, L. (2018). Los "usos y costumbres" del estado de Oaxaca en las empresas turísticas de Bahías de Huatulco. Una caracterización de la cultura organizacional de Hofstede. Investigciones Turísticas, 128-146.

Figueroa, E.; F. Pérez; L. Godínez, y R. Pérez. (2014). Importancia del Turismo en el Crecimiento Económico de México, 1989-2013. En P. María, Desarrollo Económico en el crecimiento empresarial (págs. 39-52). Bolivia: Universidad Mayor, Real y Ponticia de San Francisco Xavier de Chuquisaca.

FONATUR. (2010). Fonatur 35 años. Única historia narrada por sus fundadores $\mathrm{y}$ protagonistas. México: EDAMSA impresiones SA de CV.

FONATUR. (s/a). Agendas de competitividad de los destinos turísticos de México. México.

Hernández Licona, G. (2013). Desarrollo Económico en México. Estudios.

Hernández Rodiles, S., \& López Hernández, S. (2012). Memoria Histórica y Vida Cotidiana de los Pescadores. Alemania: Editorial Académica Española.

Kekutt, E. (Octubre-Diciembre de 2017). El desarrollo de las economías regionales bajo la mirada de un turismo. Folletos Gerenciales, XXI(4), 256-261. 
Larrosa R., J. A. (2003). El palmeral de El Che. Investigaciones Geográficas(30), 77-96. Recuperado el 15 de sep de 2016, de http://data.cervantesvirtual.com/manifestation/2 28393

López, C. B. (2007). Oaxca. Ínsula de rezagos. Crítica a sus gobiernos de razón y de costumbre. Oaxaca: Siembra.

Maldonado Ramírez, M. d. (2013). Momentos de la Construcción de la Ciudad de Oaxaca como producto turístico. Revista del Centro de Investigación. Universidad La Salle, 89-100.

Mateo Pérez, M. Á. (Otoño 2000). Sincronia. Revista de Filosofía y Letras. Obtenido de Sincronía. Revista de Filosofía y Letras: http://sincronia.cucsh.udg.mx/pobreza.htm

Moragues C., D. (2006). OEI. Obtenido de Organización de Estados Iberoamericanos: http://www.oei.es/cultura/turismodmoragues.ht $\mathrm{m}$

OMT. (2005). El turismo al servicio de los objetivos de desarrollo del Milenio. OMT. Obtenido de http://cf.cdn.unwto.org/sites/all/files/docpdf/dec laracionny-esp.pdf

OMT. (20 de septiembre de 2016). Organización mundial del Turismo. Sustainable Development of Tourism. Obtenido de Organización mundial del Turismo. Sustainable Development of Tourism:

http://sdt.unwto.org/es/content/definicion

Pérez, M. (Otoño de 2000). Sincronía. Revista de Filosófía y Letras. Obtenido de Sincronía. Revista de Filosofía y Letras: http://fuentes.csh.udg.mx/CUCSH/Sincronia/po breza.htm

Pérez, M. Á. (s.f.). Enlaces latinoamericanos para economistas. Obtenido de Enlaces latinoamericanos para economistas: http://www.oocities.org/pjabad/mateoperezsen. htm

Pipitone, U. (2007). Oaxaca: comunidad, instituciones, vanguardias. México: CIDE.
Quadratin. (20 de septiembre de 2016). Quadratin. Obtenido de Quadratin: https://oaxaca.quadratin.com.mx/descubre-inahasentamiento-precolonial-bahia-cacalutahuatulco/

Quivy. (2004). Manual de Investigación en Ciencias Sociales. (C. A. Norma, Trad.) México, México: Limusa.

Reyes, A. (Diciembre 2000). Infraestructura del turismo. Revista Construcción y Tecnología del Cemento y del Concreto AC.

Rivera R., M.A; Toledo P. A. (1998). La economía mexicana después de la crisis del peso. México: UAM-UNAM.

Rodiles, S. L., López, V. G., \& López, S. (2015). Pesca tradicional y desarrollo turístico en Bahías de Huatulco. Una lectura desde la historia oral de los pescadores locales. Investigaciones turísticas, 150-169.

S/A. (20 de septiembre de 2016). Zona arquelogica copalita. Obtenido de Zona arquelogica copalita: http://www.oaxacamio.com/huatulco/zonaarquelogicacopalita.htm Sen, A. (1998). Bienestar, justicia y mercado. (D. Salcedo, Trad.) Barcelona, España: Paidós.

Sonia, L. H. (2016). Veneración de la Santa Cruz. Bahías de Huatulco.

Talledos S., É. (2012). La imposición de un espacio: de La Crucecita a Bahías de Huatulco. Revista Mexicana de Ciencias Políticas y Sociales, 119-142.

Turismo, S. d. (9 de septiembre de 2013). Agendas de Competitividad de los destinos turísticos de México. Huatulco. Obtenido de Agendas de Competitividad de los destinos turísticos de México. Huatulco: www.sectur.gob.mx/wpcontent/uploads/2015/02/PDF-Huatulco.pdf

Turismo, S. d. (s.f.). Comisión Nacional para el Desarrollo de los Pueblos Indígenas. Obtenido de Comisión Nacional para el Desrrollo de los Pueblos Indígenas: http://www.cdi.gob.mx/ecoturismo/docs/desarr ollo_turistico_municipal.pdf 
UNESCO. (2016 de septiembre de 2016). UNESCO ¿Qué es el patrimonio cultural inmaterial? Obtenido de UNESCO ¿Qué es el patrimonio cultural inmaterial?: http://www.unesco.org/culture/ich/doc/src/0185 1-ES.pdf

Vázquez S, Valente y E. Propin F. (2004). Las relaciones regionales de la economía turística en Bahías de Huatulco, Oaxaca. Investigaciones Geográficas, Boletín del Instituto de Geografía, UNAM, 143-163.

Yáñez G., J. M. (s.f.). Estre Fuego e idolatrías: discursos y tensiones del culto a la Santa Cruz de Huatulco. México: UNAM. 